\title{
puente de Troy
}

\section{P. S. A. BERRIDGE, ingeniero}

$565-17$

En la pequeña provincia de Monmouth, en las proximidades de Gales, discurre el río Wye, sobre el que en 1876 se construyó un puente metálico, de tablero superior, dedicado al paso de una línea férrea.

El puente, llamado Troy, está constituído por un solo tramo recto de unos $45 \mathrm{~m}$ de luz. Las dos vigas maestras laterales, robustas celosías, presentan la particularidad de haberse reforzado, en su cordón inferior, con una viga cajón en cada una de estas celosías.

Debido a una mala impermeabilización y difícil acceso para la conservación en estas dos vigas cajón, se desarrolló con el tiempo una fuerte corrosión que ponía en peligro la estabilidad y resistencia del puente.

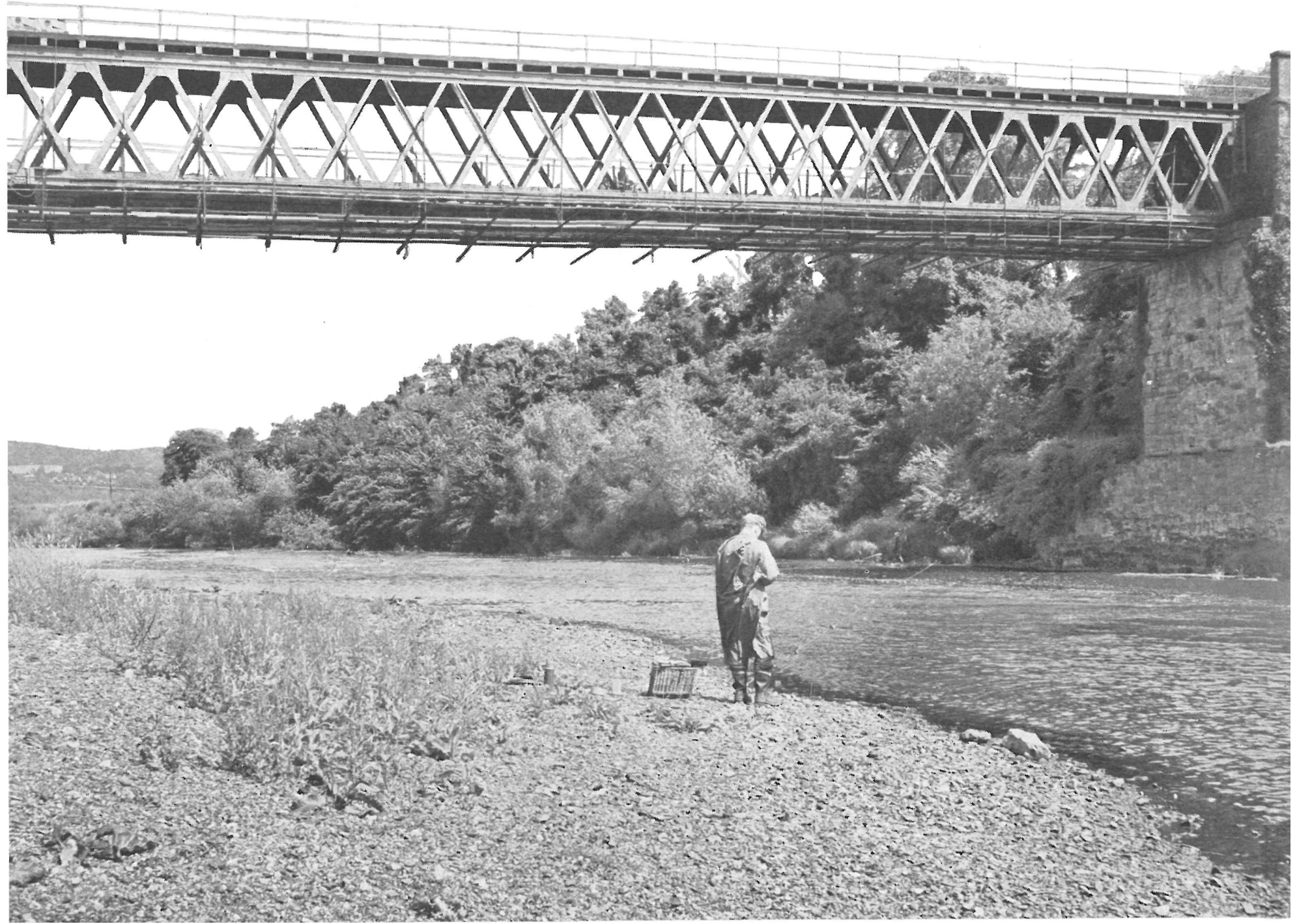




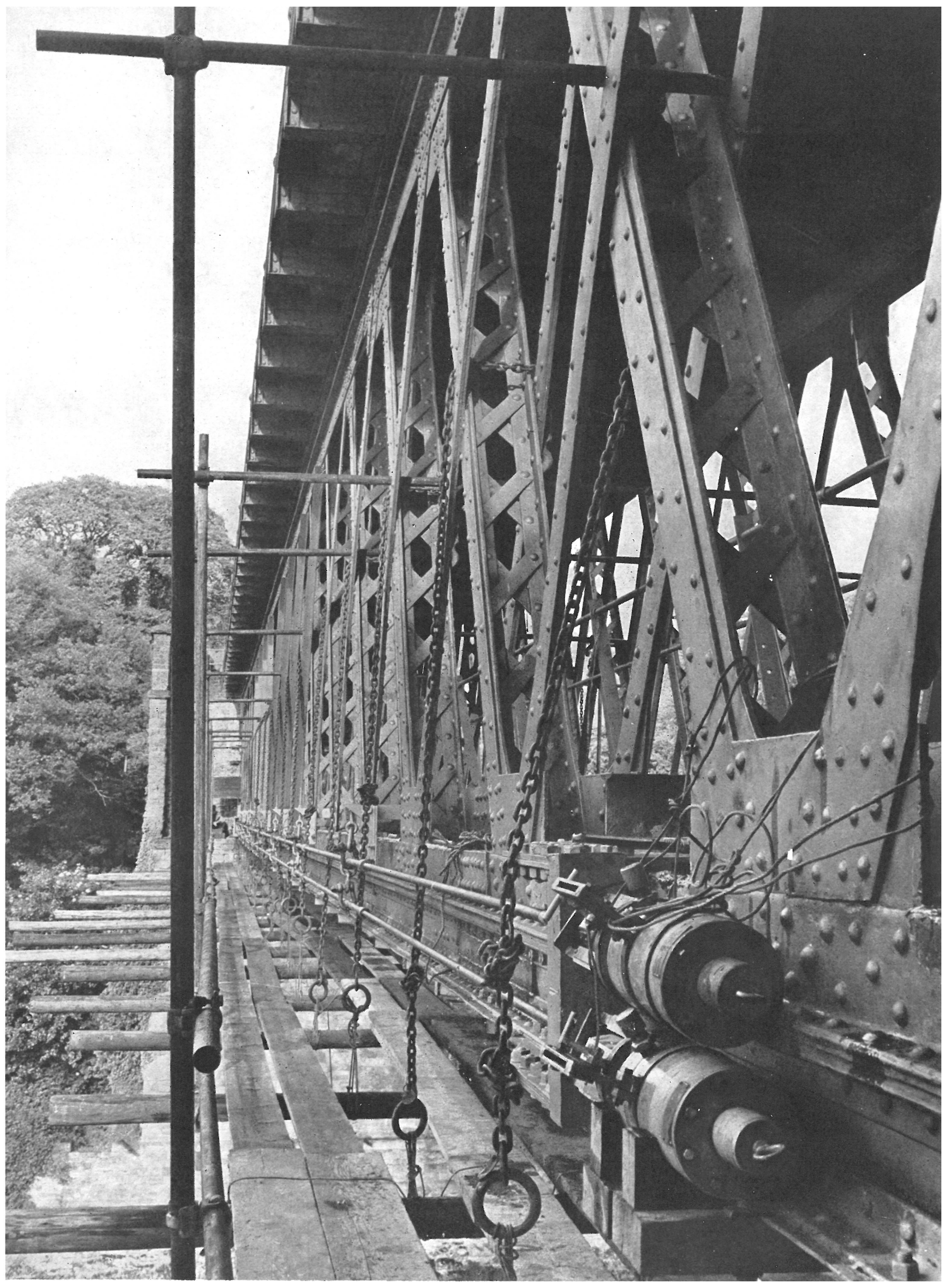



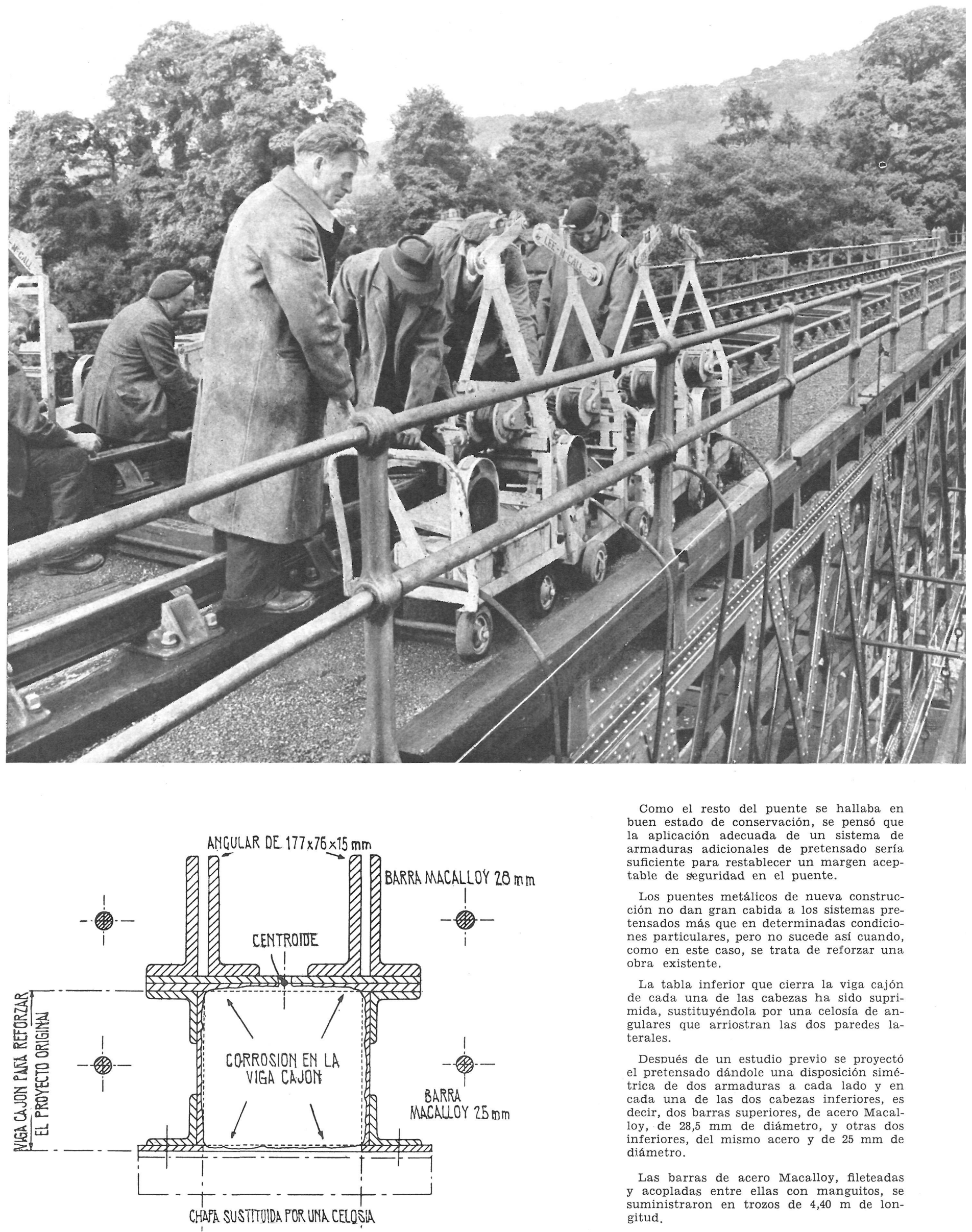

Como el resto del puente se hallaba en buen estado de conservación, se pensó que la aplicación adecuada de un sistema de armaduras adicionales de pretensado sería suficiente para restablecer un margen aceptable de seguridad en el puente.

Los puentes metálicos de nueva construcción no dan gran cabida a los sistemas pretensados más que en determinadas condiciones particulares, pero no sucede así cuando, como en este caso, se trata de reforzar una obra existente.

La tabla inferior que cierra la viga cajón de cada una de las cabezas ha sido suprimida, sustituyéndola por una celosía de angulares que arriostran las dos paredes laterales.

Después de un estudio previo se proyectó el pretensado dándole una disposición simétrica de dos armaduras a cada lado y en cada una de las dos cabezas inferiores, es decir, dos barras superiores, de acero Macalloy, de $28,5 \mathrm{~mm}$ de diámetro, y otras dos inferiores, del mismo acero y de $25 \mathrm{~mm}$ de diámetro.

Las barras de acero Macalloy, fileteadas y acopladas entre ellas con manguitos, se suministraron en trozos de $4,40 \mathrm{~m}$ de longitud. 


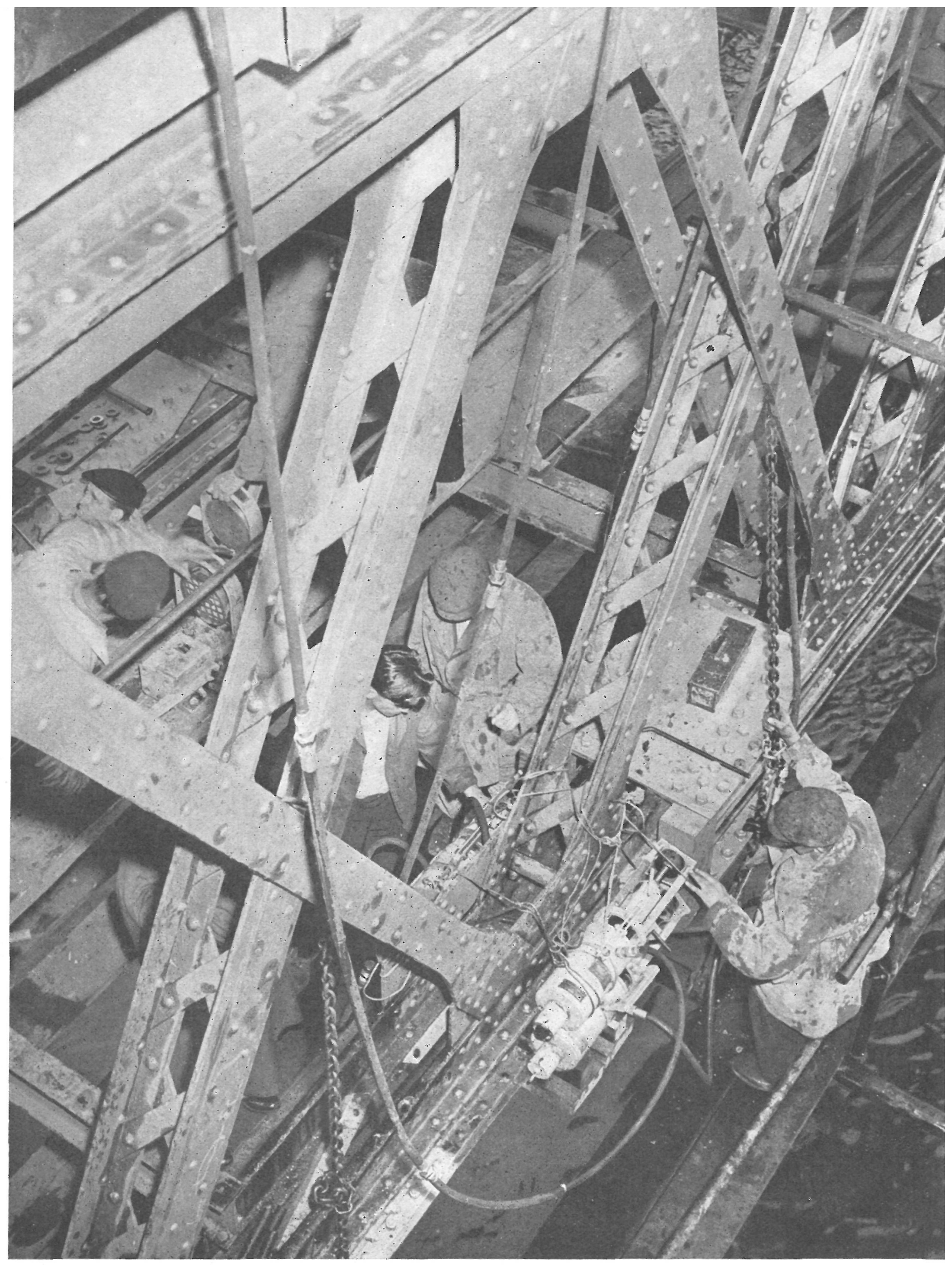

Los anclajes se colocaron a 3,10 $\mathrm{m}$ de distancia de los apoyos $\mathrm{y}$, por tanto, separados de $39 \mathrm{~m}$. La unión de los anclajes con los cordones de las dos celosías se realizó con tornillos de acero de gran resistencia. La sección de los bloques de acero utilizados para los anclajes es de $13 \times 8,8$ centímetros.

Para hacer frente a todas las operaciones de montaje y pretensado, se preparó un andamio suspendido que daba acceso a todas las partes de interés en el puente.

Las barras de acero para el pretensado se montaron suspendiéndolas provisionalmente con cables y eslingas, que después se sustituyeron por otros soportes flexibles con carácter permanente. 
Gatos en posición.

Chapas de anclaje.

Bloques de anclaje.

En el análisis del pretensado se calculó que se debía esperar un acortamiento de $5,3 \mathrm{~mm}$ en las vigas maestras. Con objeto de garantizar un movimiento libre a estas vigas, se procedió previamente a levantarlas de sus asientos sobre rodillos para preparar convenientemente estos elementos y disminuir el rozamiento.

Las ocho barras se tesaron simultáneamente con otros tantos gatos hidráulicos, tipo Lee-Mc Call. Una sola jornada de trabajo bastó para poner en tensión las ocho barras, que sufrieron un alargamiento de 10 centimetros.

Se había calculado que, con un esfuerzo de pretensado de 98,55 toneladas, las cargas de trabajo por peso propio en las zonas más débiles se reducirian de $496 \mathrm{~kg} / \mathrm{cm}^{2}$ a 63 . Para medir los acortamientos en las vigas maestras se colocaron registros amplificadores de esfera, y se pudo observar que los acortamientos reales no se diferenciaron mucho de los $5,3 \mathrm{~mm}$ que se había calculado. En la viga sur, las lecturas dieron $4,6 \mathrm{~mm}$ y sólo 4,3 $\mathrm{mm}$ la del norte. Esta diferencia se debe, probablemente, a la rigidez que proporcionan las cartelas de unión entre las almas y las cabezas de las celosías. El pretensado produjo un efecto secundario, que se tradujo por un aumento de $5,6 \mathrm{~mm}$ en la contraflecha.

Las fuerzas secundarias inducidas como consecuencia de la rigidez anteriormente referida tenían una dirección opuesta a las que da lugar la carga del peso propio; por tanto, el resultado final ha sido la reducción de estas fuerzas secundarias que ha dado lugar al pretensado.

Las barras pretensadas se han soportado con apoyos flexibles, espaciados a $4,50 \mathrm{~m}$, e independientes para cada una de las barras. Para dotar de la rigidez apropiada, al sol tar las barras de sus soportes provisionales se observó una flecha de $38 \mathrm{~mm}$, pero operando sobre el dispositivo de ajuste de los soportes permanentes se fueron elevando las barras hasta lograr $12 \mathrm{~mm}$ de contraflecha, es decir, que se puede interpretar como si se hubiesen levantado las barras unos 50 milímetros sobre su posición natural de flecha libre. Esta disposición permitió mantener las barras sin apenas cambio de posición aun bajo los efectos de las sobrecargas, para las que se ha calculado una flecha de 25 milímetros.

Terminadas las operaciones del pretensado se procedió a recubrir las barras con una cinta llamada "Densotapen para protegerlas contra los efectos de la corrosión provocada por el medio ambiente. Con objeto de poder modificar en su día el esfuerzo de pretensado, las extremidades fileteadas de las barras se recubrieron también con unas vainas de material plástico.

J. J. U.
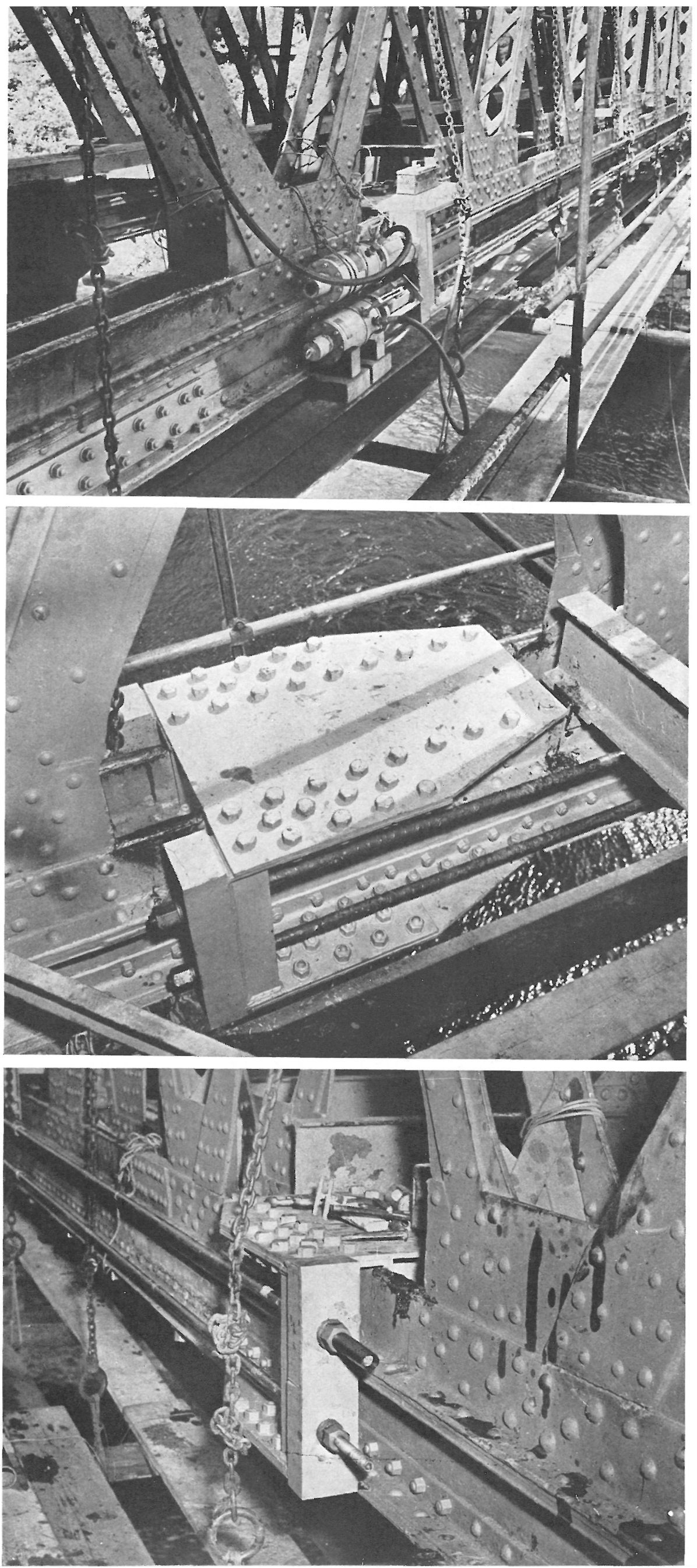